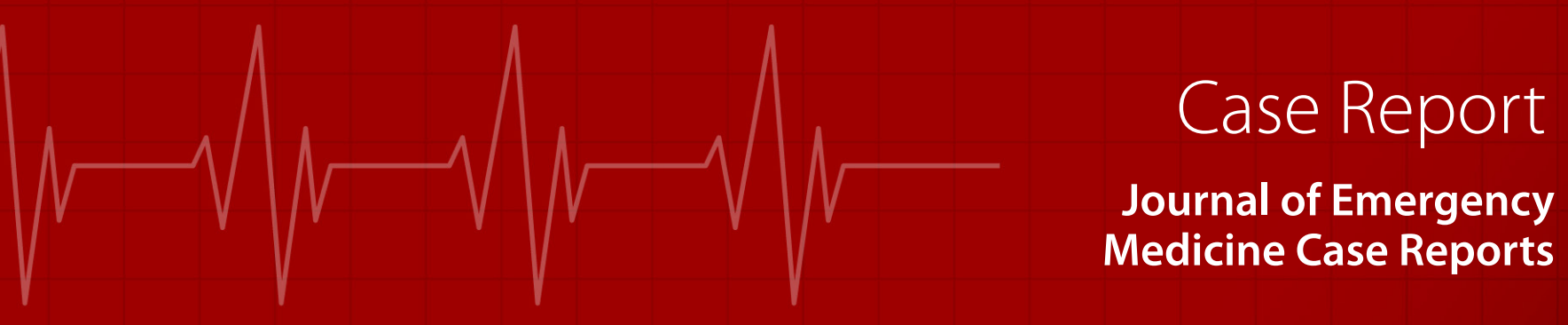

\title{
Energy Drinks Induced Atrial Fibrillation
}

Çağrı Zorlu

Department of Cardiology, Tokat State Hospital, Tokat, Turkey

\begin{abstract}
Introduction: Atrial fibrillation (AF) is most common in adulthood supraventricular arrhythmia. Some of our ways of life the occurrence of this arrhythmia possible. Recently, it is common to consume energy drinks (ED), especially among young people. Caffeine is found in energy drinks. Their adverse effects, not yet completely known, are usually considered secondary to a high concentration of caffeine. Here we focus on a particular acute cardiac complication of EDs overconsumption: atrial fibrillation.
\end{abstract}

Case report: A 23-year-old man, was referred to our emergency department for dyspnea, palpitations, and anxiety. The patient had a negative family, personal, and pharmacological history. About two hours before, he has consumed $750 \mathrm{ml}$ of ED. At admission, blood pressure was 104/62. An ECG revealed a high rate of atrial fibrillation at $162 \mathrm{bpm}$. Laboratory investigation revealed that complete blood count and routine biochemical blood levels were within normal limits..Transthoracic echocardiogram excluded the presence of cardiac disease, the left atrium was slightly dilated, as expected. According to guidelines patient was treated with propafenone and recovered a normal sinus rhythm.

Conclusion: As in our case, PAF can occur due to ED consumption, and awareness about this adverse effect of ED consumption is important.

Keywords: Arrythmia, atrial fibrillation, energy drinks

\section{Introduction}

Atrial fibrillation (AF) is most common in adulthood supraventricular arrhythmia. Some of ourways of life theoccurrence of thi sarrhythmia possible. Completely prevent AF formation from lifestyle changes not expected. However, it is possible to reduce the likelihood of AF or the frequency of attacks with some precautions. Recently, it is common to consume energy drinks (ED) especially among young people. Caffeine is found in energy drinks. Their adverse effects, not yet completely known, are usually considered secondary to an high concentration of caffeine. Here we focus on a particular acute cardiac complication of EDs overconsumption:atrial fibrillation.

\section{Case Report}

A 23-year-old man, was referred to ou remergency department for dispnea, palpitations and anxiety. The patient had a negative family, personal, and pharmacological history. About two hours before, he has consumed $750 \mathrm{ml}$ of ED. At admission, blood pressure was 104/62. An ECG revealed a high rate atrialfibrillation at $162 \mathrm{bpm}$ (Figure-1). No other physical signs were present. Laboratory investigation revealed that complete blood count and routine biochemical blood levels were with in normal limits. (Thyroid, renal, hepatic functions were normal; the hypoxia, metabolic acidosis, electrolytes imbalance, troponin increase were excluded; the toxicological results were negative.) Transthoracic echocardiogram excluded presence of cardiac disease, the left atrium was slightly dilated, as expected. According to guidelines ${ }^{1}$ patient was treated with propafenone and recovered a normal sinus rhythm (Figure-2). The patient was monitored for 6 hours, followed by sinus rhythm.

\section{Discussion}

Energy drinks contain stimulants and other substances, including large amounts of caffeine, taurine, ginseng, guarana, theophylline, sugars, vitamins, and herbs. Acute high doses have little effect on the occurrence of supraventricular arrhythmias $^{2}$. Recent ingestion of a large amount of EDs, paroxys- 


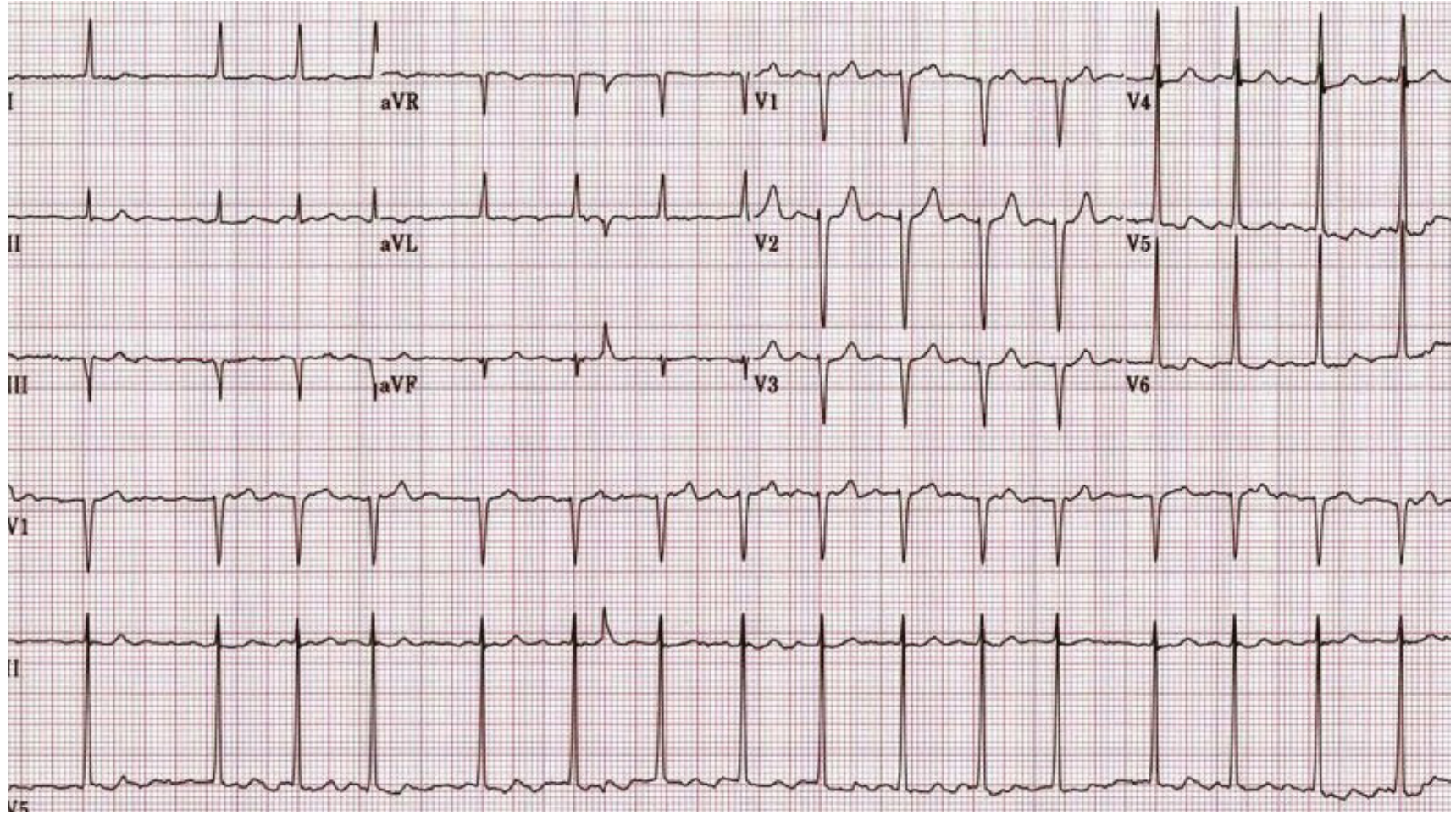

Figure 1: Atrial fibrillation after consumed energ drinks

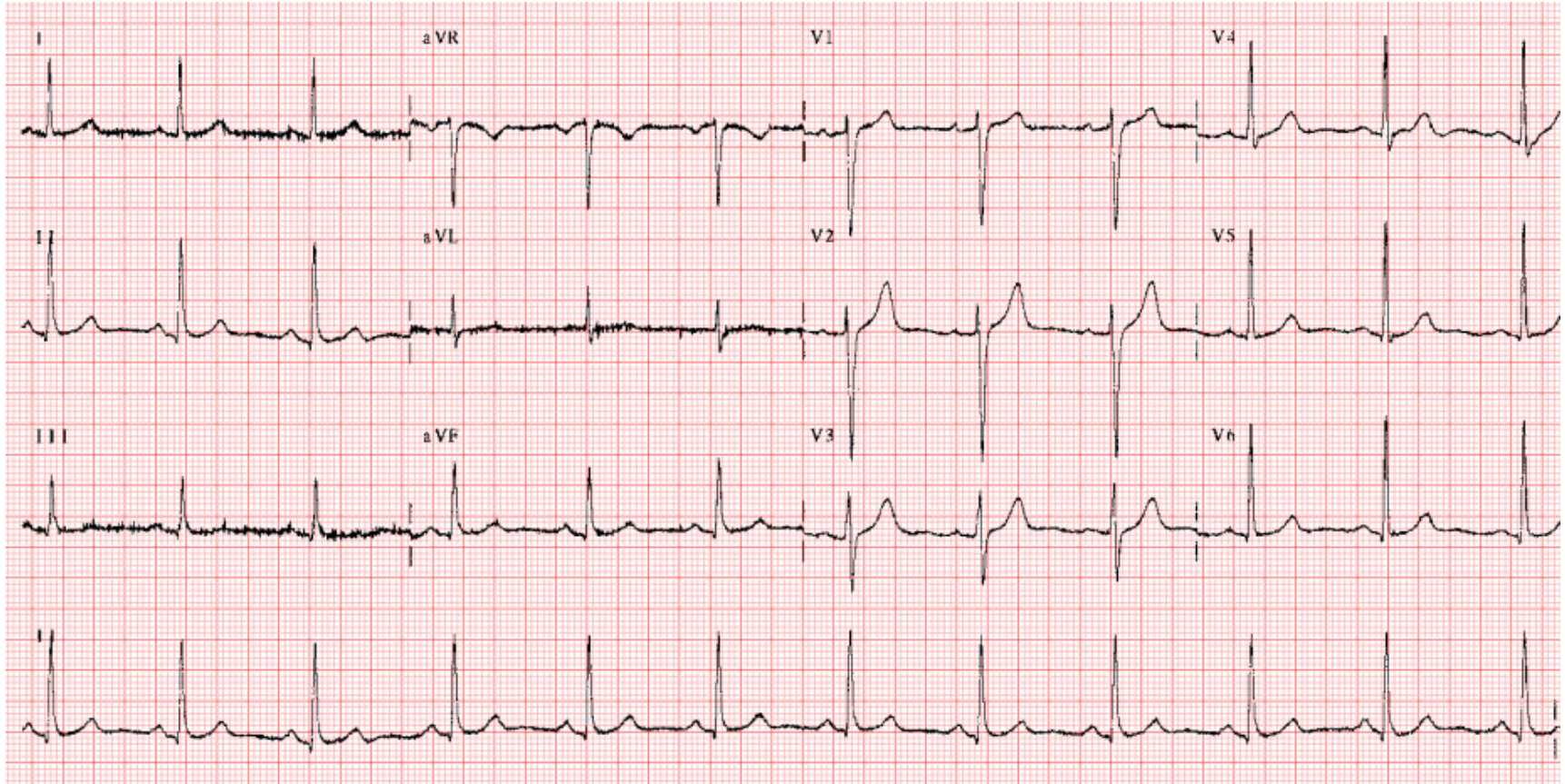

Figure 2: Treated with propafenone and recovered a normal sinus rhythm

mal $\mathrm{AF}$ at a high rate without underlying cardiac disease, and absence of laboratory abnormalities, indicating concomitant dysfunctions of other organs. Caffeine effects on cardiovascular system are well known and we want to exclude this trigger for atriall fibrillation ${ }^{3}$. A direct pathogenetic link between abuse of EDs and acute onset of high-rate AF is supported by the absence of recurrence of this complication after a complete abstinence from this beverage ${ }^{4}$.The mechanism of this phenomena is unclear, as previous studies involving high dos- es of caffeine have shown no increased risk of $\mathrm{AF}^{5}$. Caffeine is a methylxanthine compound that primarily causes neurohormonal stimulation and activation of the sympathetic nervous system by inhibitin gphosphodiesterase and adenosine $A_{1}, A_{2 A}, A_{3}$, and $A_{2 B}$ receptors and increases calcium concentration in thecytoplasm by blocking the reuptake of calcium in the sarcoplasmic reticulum ${ }^{6}$. Acute caffeine consumption was reported to increase plasma renin and norepinephrine and epinephrine levels, increasing blood pressure and heart rate ${ }^{7}$. 
It is also possible that the other ingredients and herbs in these energy drinks could potentially trigger an arrhythmia.There is no demonstration that caffeine alone increases the risk of $\mathrm{AF}^{8}$, on thecontrary, few information is available on the risk of $\mathrm{AF}$ related to other substances (i.e. taurine, maltodextrin, inositol, carnitine, creatine, guarana, ginseng, and ginkgobiloba) included in EDs. On the other hand it is unknown whether these individuals had a genetic susceptibility that predisposed to the arrhythmia.

\section{Conclusion}

As in our case, PAF can occur due to ED consumption, and awareness about this adverse effect of ED consumption is important.

\section{References}

1. 2016 ESC Guidelines for the management of atrial fibrillation developed in collaboration with EACTS. The Task Force for the management of atrial fibrillation of the European Society of Cardiology (ESC). European Heart Journal 2016 doi:10.1093/ eurheartj/ehw210.

2. Strubelt O, Diederich KW. Experimental treatment of the acute cardiovascular toxicity of caffeine. J Toxicol Clin Toxicol 1999;37:29-33.

3. Rashid A, Hines M, Scherlag BJ, Yamanashi WS, Lovallo W. The effects of caffeine on the inducibility of atrial fibrillation. J Electrocardiol 2006;39:421-5.

4. Mattioli AV, Pennella S, Manenti A, Farinetti A. Energy drink overconsumption can trigger atrial fibrillation. J Cardiovasc Med 2016.

5. Shen J, Johnson VM, Sullivan LM, et al. Dietary factors and incident atrial fibrillation: the Framingham Heart Study. Am J Clin Nutr. 2011;93:261-266.

6. Myers MG. Caffeine and cardiac arrhythmias. Ann Intern Med. 1991;114:147-150.

7. Robertson D, Frölich JC, Carr RK, et al. Effects of caffeine on plasma renin activity, catecholamines and blood pressure. $\mathrm{N}$ Engl J Med. 1978;298:181-186.

8. Mattioli AV. Caffeine and atrial fibrillation. In: V Preedy Coffee in Health and Disease prevention; Elsevier Eds 2014, pp 691697. 
